\title{
ПРОЦЕССЫ ПРЕОБРАЗОВАНИЯ В ТИРИСТОРНО-ИНДУКТИВНОЙ ЦЕПИ НЕПРЕРЫВНОГО АМПЛИТУДНОГО РЕГУЛИРОВАНИЯ ПЕРЕМЕННОГО НАПРЯЖЕНИЯ ПРИ ЕСТЕСТВЕННОЙ КОММУТАЦИИ
}

На базе тиристорных схем с фазовым управленнем в $\left[{ }^{1}\right]$ предложены цепи амплитудного регулирования переменного напряжения, а в $\left[{ }^{2}\right]$ переменного тока, форма которого близка к прямоугольной. Режим работы этих цепей основывается на подавлении четных гармоник в токе тиристора путем включения в контур замыкания постоянной и четных переменных составляющих тока тиристора сглаживающего дросселя с относительно большой постоянной индуктивностью $\left[{ }^{3}\right]$.

В целях получения многоступенчатой формы тока, близкой к синусоидальной, в Институте термофизики и электрофизики АН ЭССР разработан новый способ, основанный на управлении индуктивным накопителем энергии посредством ритмичного переключения числа витков сглаживающего дросселя. В $\left[{ }^{4}\right]$ были проанализированы процессы преобразования энергии в соответствующей новой схеме в условиях принудительной коммутации, т. е. в условиях, когда коммутационные функции всех ключей заданы.

В настоящей статье рассматривается режим естественной коммутации. Знание закономерностей преобразовательных процессов в данном случае позволит расширить область практического применения этого способа, эффективно улучшающего спектральный состав регулируемого переменного тока и напряжения.

Основные схемы тиристорно-индуктивных цепей непрерывного амплитудного регулирования переменного напряжения (рис. $1, a, \sigma, 2)$ дают возможность формировать близкий к синусоидальному ток нагрузки и могут работать как в режиме принудительной, так и в режиме естественной коммутации. В первом случае ключи должны быть полностью управляемы, а это требует применения транзисторов, запираемых тиристоров или же дополнительных элементов коммутации для запирания тиристоров. Во втором случае реализовать работу схем технически легче. Однако электромагнитные процессы в силовой цепи при естественной коммутации существенно усложняются, так как при этом задаются лишь моменты отпирания тиристоров.

С точки зрения обмена энергии между источником питания, нагрузкой и индуктивным накопителем энергии схемы на рис. 1, $a, \sigma, 2$ одинаковы. Различаются они только техническими приемами изменения участвующих в работе числа витков $w(t)$ дросселя $L$. 

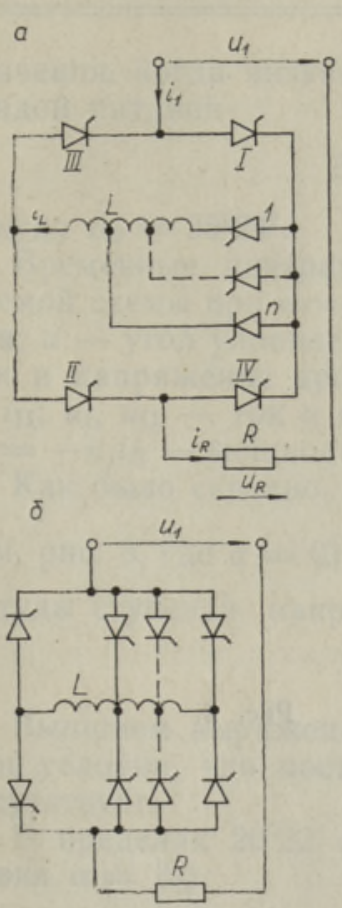

6

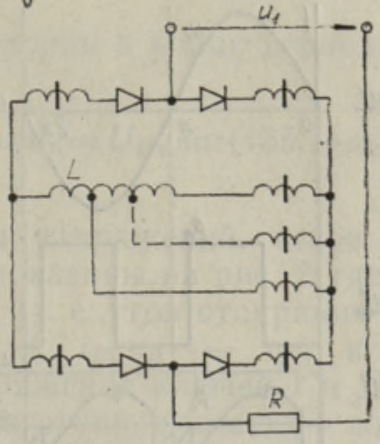

$u_{1}$

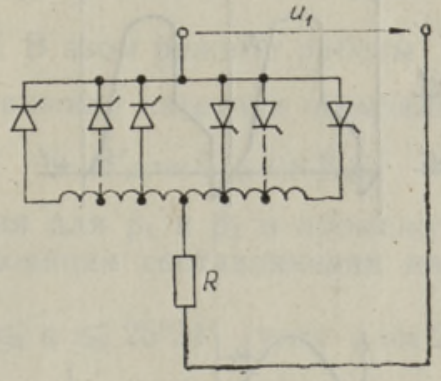

Рис. 1.

В условиях естественной коммутации тиристорные ключи могут быть заменены магнитными. В качестве примера на рис. 1, в изображена силовая схема магнитного варианта непрерывного амплитудного регулятора переменного напряжения, преобразовательные процессы в которой эквивалентны процессам в тиристорно-индуктивной схеме на рис. 1, a.

Работа всех изображенных на рис. 1 схем заключается в ритмичном изменении числа

витков $w(t)$ дросселя $L$ в каждом полупериоде переменного тока. Поэтому ограничимся рассмотрением пресбразовательных процессов в одной из них. Проанализируем схему на рис. 1, a. Здесь функции управления величиной тока выполняют тиристоры I, II, III и IV путем изменения угла управления $\alpha$, а функции управления формой тока - тиристоры $1,2 \ldots n$. Использование различных тиристоров для выполнения названных функций позволяет лучше понять сущность электромагнитных процессов.

Предположим, что схема $1, a$ работает при синусоидальном напряжении питания $u_{1}=U_{1 m} \sin \omega t$, активной нагрузке $R$, бесконечной индуктивности дросселя $L$, идеальных тиристорных ключах и двухступенчатой форме тока, т. е. при $n=2$. Кроме того, ширины и относительные амплитуды ступеней выберем из условия подавления самых низких из высших гармоник [5]. При равной ширине ступеней в каждой четверти периода углы отпирания тиристоров 1 и 2 будут равны соответственно

$$
\begin{aligned}
& \Theta_{1} \in\left\{\alpha-\frac{\pi}{4}+l \pi \mid l=0,1,2, \ldots\right\}, \\
& \Theta_{2} \in\left\{\alpha+\frac{\pi}{4}+l \pi \mid l=0,1,2, \ldots\right\} .
\end{aligned}
$$

Отметим, что при уменьшении угла управления $\alpha$ от $\pi / 2$ до некоторого предельного значения $\alpha_{\text {п }}$ преобразовательные процессы в режиме естественной коммутации протекают так же, как и в режиме принудительной $\left[{ }^{4}\right]$. Различие начинается с $\alpha<\alpha_{n}$, когда в течение отдельных этапов работы напряжение на дросселе равняется нулю, в результате чего $u_{R}=u_{1}$. Поэтому при анализе работы схемы в режиме естественной коммутации необходимо знать предельное значение угла управ- 

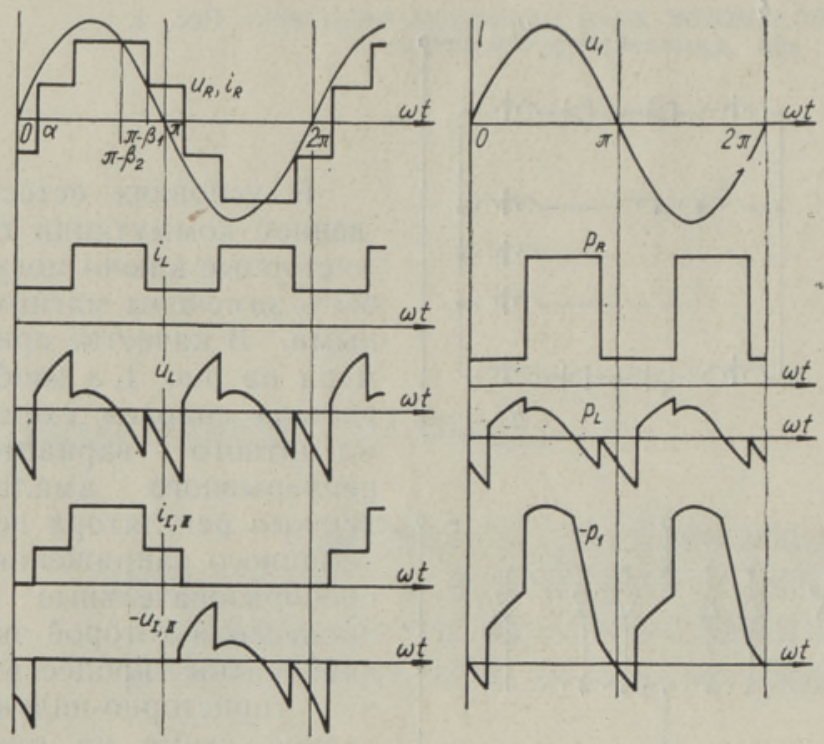

Рис. 2.
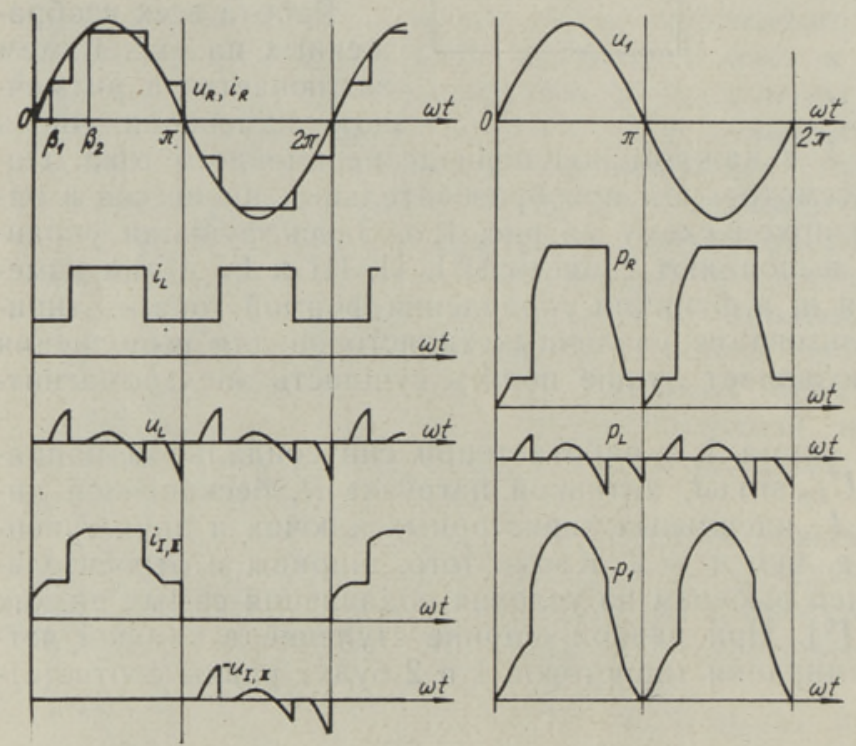

Рис. 3.

ления $\alpha_{\text {п }}$ и мгновенные значения напряжений, токов и мощностей при $\alpha<\alpha_{\text {II. }}$

Как известно из $\left[{ }^{4}\right]$, в промежутке $\alpha_{п} \leqslant \alpha \leqslant \pi / 2$ амплитуды ступеней выходного напряжения определяются по формуле

$$
B_{m}^{\prime}=B_{m} \cos \alpha
$$

где $B_{m}$ - амплитуда $m$-й ступени при $\alpha=0$ в режиме принудительной коммутацин, $B_{m}^{\prime}$ - то же при $\alpha \neq 0, m=1,2, \ldots, n$.

При двухступенчатой форме напряжения нагрузки $B_{1}=$ $=0,395 U_{R(1) m}$ и $B_{2}=0,954 U_{R(1) m}\left[{ }^{5}\right]$, где $U_{R(1) m}-$ амплитуда первой гармоники выходного напряжения, равная $0,94 U_{1 m}$.

Угол управления в рассматриваемом случае достигает предельного 
значения, когда низкая ступень в конце периода соприкасается с синусоидой питания

$$
B_{1} \cos \alpha_{\mathrm{II}}=U_{1 \mathrm{~m}} \sin \left(135^{\circ}+\alpha_{\mathrm{II}}\right)
$$

откуда $\alpha_{\Pi}=25^{\circ} 24^{\prime}$.

Временные диаграммы напряжений, токов и мощностей анализи-

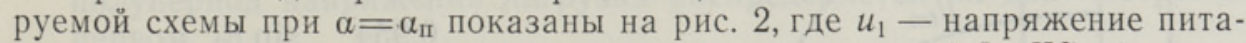
ния; $\alpha-$ угол управления (т. е. угол отпирания ключей $\mathrm{I}-\mathrm{IV}) ; i_{L}, u_{L}-$ ток и напряженне дросселя; $i_{R}, u_{R}$ - ток и напряжение нагрузки; $i_{\mathrm{I}}, i_{\mathrm{II}} ; u_{\mathrm{I}}, u_{\mathrm{II}}-$ ток и напряжение ключей I и II; $p_{R}=u_{R} i_{R}, p_{L}=u_{L} i_{L}$, $p_{1}=-u_{1} i_{R}-$ мощности нагрузки, дросселя и питания.

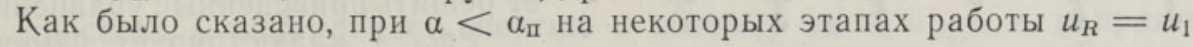
(см. рис. 3 , где $\alpha=0$ ). В этом режиме работы $\left(-\frac{\pi}{2 n} \leqslant \alpha \leqslant \alpha_{\Pi}\right)$ амплитуды ступеней напряжения нагрузки определяются соотношением

$$
B_{m}^{\prime}=U_{1 m} \sin \beta_{m} \text {. }
$$

Выпишем выражения для $\beta_{1}$ и $\beta_{2}$ в промежутке $-45^{\circ} \leqslant \alpha \leqslant 25^{\circ} 24^{\prime}$ при условии, что постоянная составляющая напряжения дросселя $u_{L}$ отсутствует.

В пределах $20^{\circ} 22^{\prime} \leqslant \alpha \leqslant 25^{\circ} 24^{\prime}$ (угол $\alpha=20^{\circ} 22^{\prime}$ определен из условия $\left.\alpha=\beta_{1}\right)$ :

$\frac{\sqrt{2}}{2} \sin \alpha+\left(2+\frac{\sqrt{2}}{2}\right) \cos \alpha-\cos \beta_{1}-\left(\frac{\pi}{4}+\alpha+\beta_{1}\right) \sin \beta_{1}-\frac{\pi}{2} \sin \beta_{2}=0$;

в пределах $15^{\circ} 05^{\prime} \leqslant \alpha \leqslant 20^{\circ} 22^{\prime}$ (угол $\alpha=15^{\circ} 05^{\prime}$ определен из условия $\left.\beta_{2}=\frac{\pi}{4}+\alpha\right)$ :

$$
\frac{\sqrt{2}}{2} \sin \alpha+\left(1+\frac{\sqrt{2}}{2}\right) \cos \alpha-\left(\frac{\pi}{4}+2 \alpha\right) \sin \beta_{1}-\frac{\pi}{2} \sin \beta_{2}=0 ;
$$

в пределах $-21^{\circ} 09^{\prime} \leqslant \alpha \leqslant 15^{\circ} 05^{\prime}$ (угол $\alpha=-21^{\circ} 09^{\prime}$ определен из условия $\left.|\alpha|+\beta_{1}=\pi / 4\right)$ :

$$
\begin{gathered}
\sqrt{2} \sin \alpha+\cos \alpha-\left(\frac{\pi}{4}+2 \alpha\right) \sin \beta_{1}+\cos \beta_{2}- \\
-\left(\frac{3 \pi}{4}+\alpha-\beta_{2}\right) \sin \beta_{2}=0
\end{gathered}
$$

в пределах $-24^{\circ} 01^{\prime} \leqslant \alpha \leqslant-21^{\circ} 09^{\prime}$ (угол $\alpha=-24^{\circ} 01^{\prime}$ определен из условия $\left.\alpha=-\beta_{1}\right)$ :

$$
\begin{gathered}
\frac{\sqrt{2}}{2} \sin \alpha+\left(1+\frac{\sqrt{2}}{2}\right) \cos \alpha-\cos \beta_{1}-\left(\alpha+\beta_{1}\right) \sin \beta_{1}+\cos \beta_{2}- \\
-\left(\frac{3 \pi}{4}+\alpha-\beta_{2}\right) \sin \beta_{2}=0
\end{gathered}
$$

в пределах $-45^{\circ} \leqslant \alpha \leqslant-24^{\circ} 01^{\prime}$ (угол $\alpha=-45^{\circ}$ определен из условия $\left.\beta_{2}=\pi / 2\right)$ :

$$
\cos \left(\frac{3 \pi}{4}+\alpha\right)-\cos \beta_{2}+\left(\frac{3 \pi}{4}+\alpha-\beta_{2}\right) \sin \beta_{2}=0 .
$$




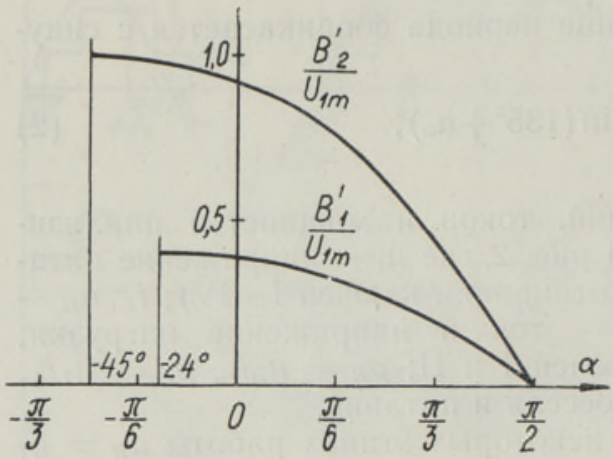

Рис. 4.

Поскольку относительные амплитуды ступеней определяются числом витков дросселя $L$, включенных в данный момент в диагональ выпрямленного тока, то $\beta_{1}$ и $\beta_{2}$ связаны соотношением

$$
\frac{0,954}{0,395}=\frac{\sin \beta_{2}}{\sin \beta_{1}}
$$

использование которого позволяет решить приведенные уравнения.

Рис. 4 иллюстрирует изменение амплитуды ступеней во всем диапазоне регулирования. При $\alpha=-24^{\circ}$ исчезает первая ступень, при $\alpha=-45^{\circ}-$ вторая. В интервале $-\pi / 2 \leqslant \alpha \leqslant-\pi / 4$ форма напряжения нагрузки совпадает с синусоидой.

Временные диаграммы мощностей на рис. 2 и 3 показывают процесс преобразования энергии в режиме естественной коммутации. В течение $\alpha \leqslant \omega t \leqslant \pi-\beta_{2}$ нагрузка потребляет не всю энергию источника питания, остаток ее накапливается в дросселе. В промежутке $\pi-\beta_{2} \leqslant$ $\leqslant \omega t \leqslant \pi$ нагрузка питается как от источника питания, так и от дросселя, а в интервале $\pi \leqslant \omega t \leqslant \pi+\alpha-$ только от дросселя. Остаток накопленной энергии возвращается в источник питания.

Интересно отметить, что по мере уменьшения угла $\alpha$ влияние, оказываемое дросселем на процесс преобразования энергии, становится все меньше и при $\alpha=-\pi / 2 n$ исчезает, так как ток нагрузки достигает синусондальной формы и $u_{L}=0$.
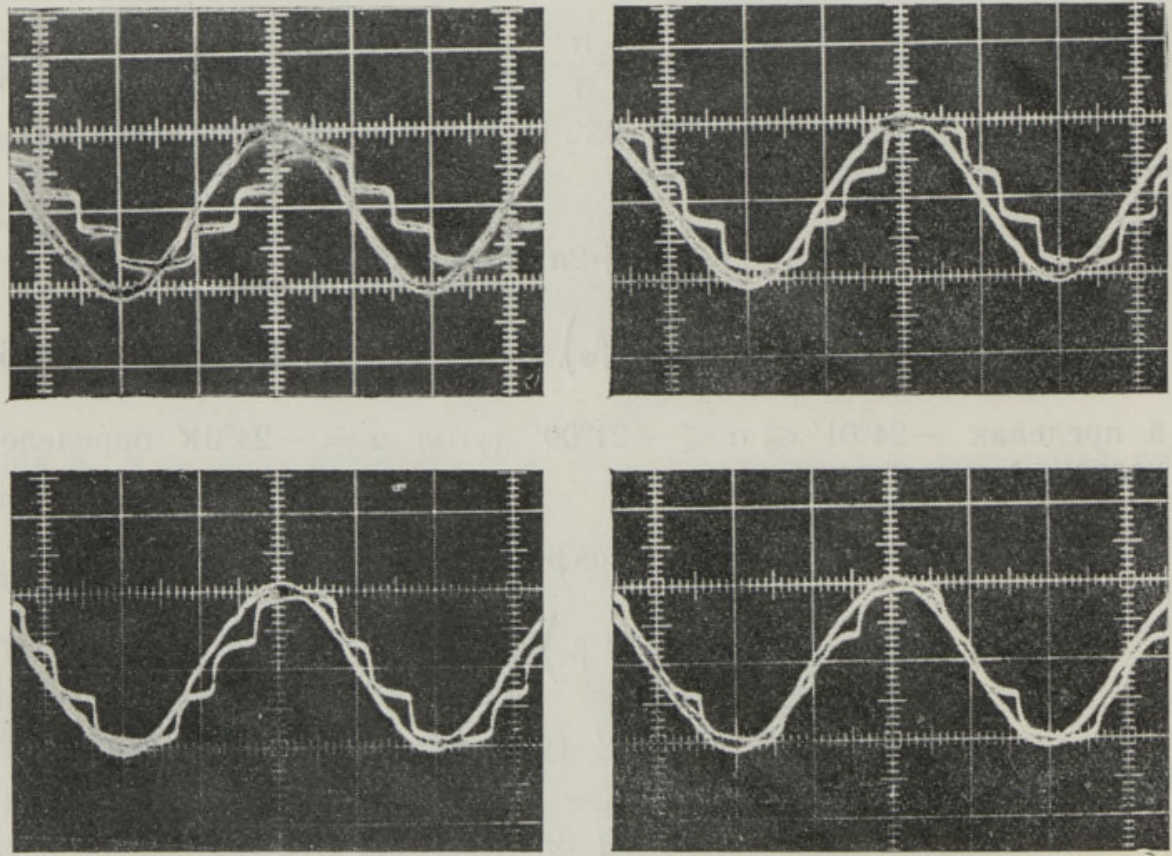

Рис. 5. 
Хотя в статье мы ограничились анализом идеализированного случая, осциллограммы напряжений питания $u_{1}$ и нагрузки $u_{R}$ лабораторного макета при $n=2$ и $\alpha=\operatorname{var}$ (рис. 5) подтверждают совпадение процессов в идеализированных и реальных цепях.

Анализ, проведенный при $n>2$, показал, что характер преобразовательных процессов не зависит от количества ступеней и что каждый полупериод напряжения нагрузки распределяется на два интервала. В первом

$$
\alpha \leqslant \omega t \leqslant \pi-\arcsin \frac{B_{n}^{\prime}}{U_{1 m}}
$$

дроссель $L$ накапливает энергию и поэтому $u_{R} \leqslant u_{1}$, т. е. напряжение резистивной нагрузки не превышает напряжения питания. Во втором интервале

$$
\pi-\arcsin \frac{B_{n}^{\prime}}{U_{1 m}} \leqslant \omega t \leqslant \alpha+\pi
$$

дроссель отдает накопленную энергию и потому $u_{R} \geqslant u_{1}$.

Изложенное позволяет сделать вывод, что при переходе тиристорноиндуктивного непрерывного амплитудного регулятора переменного напряжения из режима принудительной коммутации в режим естественной форма кривой тока усложняется, если значение угла управления $\alpha$ ниже некоторого предельного его значения $\alpha_{\Pi}$, но спектральный состав тока в то же время улучшается. Поэтому предлагаемые схемы тиристорно-индуктивных амплитудных регуляторов с естественной коммутацией заслуживают широкого применения на практике.

\section{ЛИТЕРАТУРА}

1. Emanuel-Eigeles, A., Erlicki, M. S. New aspects of power factor improvement. P. II. - IEEE Trans. Industry and General Applications, 1968, v. IGA-4, N 4, p. $447-455$.

2. Lepp, V. R., Sibgatulin, K. M., Cherkasov, J. N. Current stabilizer having a saturable reactor in the mode of forced magnetization. - United States Patent, 1974, N 3845380 , Oct. 29.

3. О я в э эр М., С ар в В. Процессы преобразования в тиристорном регуляторе переменного напряжения с подавленными четными гармониками тока. - Изв. АН ЭССР, Физ. Матем., 1976 , т. 25 , № 4, с. $392-397$.

4. О я в э э р М., С а р в В. Анализ преобразовательных процессов в индуктивно-ключевой цепи непрерывного амплитудного регулирования переменного напряжения. - Изв. АН ЭССР, Физ. Матем., 1977, т. 26, № 3, с. 297-303.

5. Тонкаль В. Е., Липковский К. А., М ельничук Л. П. Способы улучшения качества выходного напряжения автономных инверторов. - Препринт-49 ИЭД АН УССР. Киев, 1972.

Институт термофизики и электрофизики Академии наук Эстонской ССР

Поступила в редакцию 19/IX 1977 
Maire OJAVEER, V. SARV

\section{MUUNDUSPROTSESSID VAHELDUVPINGE AMPLITUUDI SUJUVALT REGULEERIVAS INDUKTIIVSES TURISTORAHELAS VABAKOMMUTATSIOONI KORRAL}

Artiklis on esitatud vahelduvpinge amplituudi sujuvalt reguleerivate induktiivsete türistorahelate skeemivariandid, mis võimaldavad saada mitmeastmelist siinuselisele lähedast koormusvoolu. On määratud muundusprotsessi seaduspärasused vabakommutatsiooni korral ning neid illustreeritud pingete, voolude ja võimsuste ajaliste diagrammidega kaheastmelise voolu juhul.

Maire OJAVEER, V. SARV

\section{CONVERSION PROCESSES IN THE THYRISTOR-INDUCTIVE CIRCUIT OF ALTERNATING VOLTAGE CONTINUOUS AMPLITUDE-REGULATION IN CONDITIONS OF NATURAL COMMUTATION}

Some basic configurations of thyristor-inductive circuits of alternating voltage continuous amplitude-regulation for obtaining a multistep current wave-form near to sinusoidal, are presented. Conversion processes in conditions of natural commutation are described and the wave-forms of voltages, currents and powers in the case of two-step load current wave-form are presented. 